\title{
Política exterior y estados periféricos. Análisis de la transformación de la identidad del Ecuador
}

Mauricio Jaramillo Jassir**

\section{RESUMEN}

El artículo tiene por objeto analizar el cambio de identidad en la política exterior ecuatoriana, apoyándose en la necesidad conceptual por establecer un vínculo entre lo interno y lo externo. La hipótesis de trabajo consiste en que entre 2008 y 2011, Ecuador transformó su identidad dotándose de un nuevo rol en el sistema internacional, resultado del proceso refundacional. Su identidad consistió en buscar un equilibrio regional, donde pudiese influir en ciertos temas, sin alinearse con otros Estados y reduciendo lo que percibía como vulnerabi- lidades. Para demostrarlo, el texto se divide en tres partes. En primer lugar, se analizan los desafíos de la política exterior como campo de estudio para los llamados Estados periféricos; en segundo lugar, se aborda la dimensión exterior de la transformación ecuatoriana durante el gobierno de Rafael Correa, sobre todo, entre 2008 y 2011 y, finalmente, se esbozan algunas ideas sobre la manera en que debe ser interpretado el cambio de identidad ecuatoriana.

Palabras clave: política exterior ecuatoriana, identidad de rol, Ecuador, construcción del Estado-nación, tercer mundo

\footnotetext{
** Agradezco los comentarios valiosos de Andrés Molano Rojas, Arlene Tickner, Ralf Leiteritz y Dianne TawseSmith.

* Doctor en ciencia política de la Universidad de Toulouse, magíster en geopolítica de la Universidad París 8 y en relaciones internacionales de Sciences Po Toulouse e internacionalista del Rosario. Fue asesor del Secretario General de Unasur y es profesor de carrera en la Facultad de Ciencia Política, Gobierno y Relaciones Internacionales de la Universidad del Rosario, Bogotá (Colombia). [mauricio.jaramilloj@urosario.edu.co]; [https://orcid.org/0000-00016190-1054].

Recibido: 23 de agosto de 2019 / Modificado: 17 de enero de 2020 / Aceptado: 29 de enero de 2020

Para citar este artículo:

Jaramillo Jassir, M. (2020). Política exterior y Estados periféricos. Análisis de la transformación de la identidad del Ecuador. OASIS, 32, pp. 153-175.

Dor: https://doi.org/10.18601/16577558.n32.10
} 


\section{Foreign policy and peripheral states. Ecuadorian identity transformation analysis}

\section{ABSTRACT}

This article aims to analyze the Ecuadorian foreign policy in terms of identity, through the study of the dynamics of domestic and foreign affairs. The hypothesis lies in the idea that Ecuadorian transformation (2008-2011) had an impact on its international performance. During Rafael Correa's administration, Ecuador's identity relied on the regional balance of power, the non-alignment and the reduction of what the country perceived as vulnerabilities. The article is divided in three parts. First, foreign policy analysis of peripheral states is assessed, in order to identify some limitations that must be overcome. Second, Rafael Correa`s Citizenship Revolution foreign policy is explored. Finally, we addressed the way in which Ecuadorian identity transformation must be conceived.

Key words: Ecuadorian foreign policy, role identity, Ecuador, state-building, third world

\section{INTRODUCCIÓN}

¿Cuál fue el impacto del proceso refundacional del gobierno de Rafael Correa en la política exterior ecuatoriana? Se trata de una pregunta fundamental para entender el vínculo mutable entre lo interno y lo externo y en la exploración de la identidad de rol de algunos Estados periféricos o del sur global ${ }^{1}$. El propósito central del presente artículo consiste en demostrar que el cambio exterior ecuatoriano, puede ser interpretado como la consecuencia de un cambio en la identidad, que no debe ser adjudicada exclusivamente a ese gobierno, pues en el pasado reciente, en especial en la segunda mitad de los noventa, Ecuador atravesó por un período crítico que provocó trasformaciones en la autopercepción y en la de terceros. Este esquema interpretativo, que se puede considerar como constructivista, pues alude a la intersubjetividad, se complementa con el realismo subalterno, pues se apela a la incidencia determinante del proceso inacabado del Estado-nación en su política exterior.

La pretensión de este trabajo consiste en ahondar en la transformación de la identidad ecuatoriana partiendo de las siguientes hipótesis de trabajo:

- En los Estados de la periferia que atraviesan por transiciones resulta imposible omitir que la política exterior es una extensión de la dinámica doméstica. Esto se puede explicar a partir de la interacción de la política interna-externa de la que habla Andrew Moravsick y en la que se pone de relieve la forma como el proceso

\footnotetext{
1 Para efectos de este artículo se le denominará como perteneciente al Tercer Mundo según la nomenclatura del realismo subalterno. También se alude a los Estados pequeños y periféricos, pues en la mayoría de estudios sobre política exterior se apela a esas denominaciones.
} 
democratizador interno tiene un efecto determinante en la proyección de los Estados. La política exterior no debe equipararse únicamente a una estrategia, táctica o al interés nacional, pues la misma es el resultado de un proceso de negociación constante en el que participan cada vez más actores de la sociedad (Moravcsik, 1997, p. 519) (Moravcisk, 1993, pp. 1517). Este enfoque liberal no es incompatible con las teorías sobre la concepción nacional del rol, pues en determinados contextos la misma sociedad civil participa de dicha construcción.

- El proceso inacabado de construcción de Estado-nación condiciona la política exterior de actores que, como Ecuador, constantemente apelan a proyectos para la refundación del Estado y, paralelamente, para la consolidación de la democracia (Ayoob, 1995).

- Los costos de la autonomía para el realismo periférico son determinantes a la hora de explicar el comportamiento exterior de los Estados de la periferia. Según (Schenoni y Escudé, 2016, p. 4) el alcance de una autonomía plena suele derivar en mayores costos para los Estados que tienen reivindicaciones nacionalistas, pues asumen las sanciones de potencias y dejan de recibir beneficios del alineamiento. No obstante, el caso ecuatoriano sirve para contradecir dicho argumento.

- Los cambios de roles en la política exterior de los Estados pequeños no solo se explican por razones estructurales, propias de constreñimientos del sistema internacio- nal, sino por las interacciones simbólicas tal como lo plantea Thies (2017, p. 663) en una suerte de síntesis entre el constructivismo y el neorrealismo.

A partir del caso ecuatoriano, y partiendo de tales postulados, se pretende entender qué origina una alteración de la identidad de rol de los Estados periféricos, cuáles son los efectos y qué patrones de observación permiten constatarla. Tradicionalmente, se asumía que las principales motivaciones para la conducta exterior de dichos actores residían en la seguridad y en la supervivencia, fiel reflejo de la lectura realista o neorrealista de las relaciones internacionales (Morgenthau, 1993; Waltz, 1979), condicionantes mayores de los análisis en política exterior. No obstante, se debe sobrepasar tal concepción que condiciona y minimiza sus intereses. Robert Keohane argumentó que para entender el rol de los Estados pequeños, había que ir más allá de sus preocupaciones en seguridad, pues era vital abordar la propia percepción del rol. Esto quiere decir que no siempre el rol o los intereses de un Estado pequeño dependen de los constreñimientos sistémicos o estructurales (Keohane, 1969, p. 295). En este mismo sentido, el realismo periférico de Carlos Escudé desarrolló tres críticas contra el neorrealismo en su análisis estructural de la política exterior de los Estados pequeños o periféricos, al asumirlos como una unidad de análisis sin reparo por las diferencias de capacidades entre uno y otro, la preeminencia de la seguridad para la definición del interés nacional y la anarquía como principio ordenador del sistema internacional (Schenoni y Escudé, 2016, p. 2). 
Ecuador es poco estudiado en términos de política exterior. Por lo general, la literatura sobre la concepción nacional de rol ha sido estudiado en casos paradigmáticos como Argentina, Brasil, Colombia, Chile (Bernal, 2015; Giacalone, 2012) o Cuba (Noesselt, 2014). Ecuador, por su parte, ha sido objeto de numerosos análisis en cuanto al populismo, la movilización indígena o la instabilidad, pero son más escasos aquellos que versen sobre su política exterior.

Algo similar se puede decir de los diez años de gobierno de Rafael Correa, pues inspiró numerosas reflexiones sobre el carácter autoritario o democrático del proceso, pero el aspecto exterior ha sido abordado con menor profusión, pues se considera como un proceso netamente interno.

De igual forma, el artículo resulta aún más pertinente tomando en cuenta la importancia creciente de los Estados periféricos en la agenda internacional, gracias a un cierto nivel de especialización en temas relevantes para el sistema internacional, o al menos un liderazgo regional que se puede proyectar por la vía del esquema de la cooperación Sur-Sur. Dichos Estados, están más interesados en fungir como líderes que como potencias, pues pretenden una interlocución para ciertos temas, sin asumir una responsabilidad y careciendo de atributos de poder. Por ende, a lo largo del texto, se observará que, durante el gobierno de Rafael Correa,
Ecuador apostó por una influencia regional con el apoyo de pares, pero sin asumir ninguna responsabilidad de potencia, pues en poco podía alterar su posición estructural.

El estudio compagina distintos enfoques teóricos en política exterior que explican aspectos de la proyección ecuatoriana que una sola aproximación conceptual o teórica no puede abarcar.

\section{LOS POSIBLES VACÍOS PARA ENTENDER LA POLÍTICA EXTERIOR DE ESTADOS PERIFÉRICOS}

La política exterior puede ser entendida como un subcampo o subdisciplina de las relaciones internacionales, razón por la cual se articula a partir de conceptos, enfoques o teorías de rango medio. Uno de los debates fundamentales de las relaciones internacionales consiste, precisamente, en determinar el vínculo entre la política interna y la exterior. Para realistas o neorrealistas como Kenneth Waltz, John Mearsheimer, Hedley Bull y Hans Morgenthau, existe una clara diferenciación entre ambos. En últimas, la política internacional ${ }^{2}$ termina condicionando la proyección de un Estado, lo cual se podría entender por el principio de la "primacía de la política exterior" (Rynning \& Guzzini, 2001, p. 3) propio del siglo XIX y aplicable a lo largo del xx.

2 La política internacional consiste en la interacción entre todos los actores del sistema internacional, mientras que la política exterior consiste en el conjunto de acciones que un actor emprende hacia el sistema. Se trata de nociones que muchas veces se equiparan, pero cuya diferenciación es esencial en cuanto a los niveles de análisis en las relaciones internacionales. 
Para Morgenthau el interés nacional definido en términos de poder orienta la política exterior. Su funcionamiento reside en la razón de Estado, y en nada tiene que ver el perfil psicológico del estadista, ni sus motivaciones personales o ideológicas (Morgenthau, 1993, p. 5). Waltz, por su parte, sentenció que una teoría de política internacional, tal como la concibió, no podía ser entendida como marco de referencia para la política exterior (Waltz, 1979, p. 121).

En la otra orilla, en la corriente liberal, aparece el célebre esquema de Robert Putnam que denominó “juego a dos niveles" (Puntman, 1988, pp. 430-432), basado en la presunción de que el jefe de Estado o de gobierno, responde a dos escenarios de manera simultánea (interno y exterior). Asimismo, Puntman propone una clasificación a partir de una revisión de la literatura, sobre el vínculo entre lo interno y lo exterior que aclara el panorama y permite entender dónde se pueden hallar vacíos o temas para complementar:

a. Los estudios que siguen la línea de James Rosenau, son en particular influyentes en el campo de la política exterior comparada. Se privilegia el estudio de las reacciones de los Estados frente a determinados estímulos en el área internacional (Rosenau, 1968).

b. El enfoque que privilegia la integración regional como campo de la política exterior y recientemente centrado en la gobernanza. En esta lectura, los trabajos de Karl Deutsch y de Ernst Haas, inspirados en el modelo de integración de Europa, son paradigmáticos (Deutsch, 1957; Haas, 1958).

c. Una serie de trabajos que abordan la política exterior como una política pública, por lo que privilegian una lectura burocrática, tomando como principal referente los modelos de Graham Allison, centrados en la correlación de fuerzas entre los actores dentro de los Estados (Allison \& Zelikow, 1999) y la complejidad del proceso de toma de decisiones por la participación de varios sectores del Estado y de la sociedad.

d. Peter Katzenstein y Stephen Krasner analizaron los vínculos entre la economía política internacional y la forma en que los Estados buscan hacer compatible su política exterior con esta (Katzenstein, 1976; Krasner, 1977).

A esa clasificación de Puntman, habría que añadir dos corrientes. Una interpretación de la identidad, desde el constructivismo en la que el lenguaje (Kowert, 1998, p. 105), los actos discursivos y la interacción simbólica -intersubjetividad- (Onuf, 1998) son esenciales para entender el comportamiento exterior de los Estados.

De igual forma, se deben sumar los enfoques que desde la periferia han surgido para explicar el comportamiento de Estados pequeños, subalternos, periféricos, del tercer mundo, o del sur global. Etiquetas que varían según autores o escuelas. En algunos países del sur global, se reivindican teorías que desafían la hegemonía académico-científica que, a la larga, refleja la división del mundo entre centro y pe- 
riferia. Algunos estudios revelan los intentos serios por establecer una epistemología o teorías de las relaciones internacionales que respondan a esa necesidad, poco suplida (Tickner, 2008; Acharya, 2014; Neuman, 1998; Ayoob, 2002).

El realismo subalterno plantea que:

[...] Las Relaciones Internacionales reflejan y reproducen la desigualdad presente en la distribución de capacidades en el sistema internacional. El poder traducido en la dominación en la esfera de la producción y reproducción de conocimiento. La dominación en el ámbito del conocimiento legitimará, por tanto, la desigualdad en el sistema internacional debido a esas capacidades bajo el control de Estados dominantes y de sociedades que añaden a ese control el poder blando y el poder duro. Romper el monopolio de ese control del conocimiento, requiere que consideremos seriamente este intento [del realismo subalterno] de presentar alternativas conceptuales a las teorías que dominan las Relaciones Internacionales (Ayoob, 2002, p. 27).

A esta denuncia sobre la primacía de lo occidental o del primer mundo, se suma la necesidad de tener análisis más rigurosos sobre el impacto de lo interno en la política exterior. Fearon (1998, p. 290) propuso una revisión detallada sobre el número de estudios dedicados a observar los factores internos, como explicativos de la política exterior. Entre 1986 y 1996, de un total de 193 resúmenes de artículos publicados en la revista International Organization, dedicados a la política exterior, Fearon halló dos tercios que invocan lo interno como variable independiente o interviniente de la política exterior. Sin embargo, planteó la necesidad de avanzar en una teoría de lo interno, para dar cuenta de la política exterior, sugiriendo que aun con la abundante literatura dedicada al tema, subsistían dos preguntas inconclusas: ¿En qué consiste el análisis interno de la política exterior? ¿Qué elementos deben tenerse en cuenta para determinar que una decisión de política exterior obedece a dinámicas domésticas?

Con este panorama, se puede entender la complejidad que supone el estudio de la política exterior de los latinoamericanos. Considerables o no en la órbita de occidente, sus características internas, que se desprenden del proceso inacabado en la construcción del Estado-nación, hacen necesaria la tarea de incorporar, en los análisis de política exterior, variables internas.

De otra parte, los estudios sobre política exterior latinoamericana son numerosos. La literatura durante la guerra fría estuvo fuertemente permeada por la idea de autonomía o alineación, frente a los dos grandes modelos político-económicos del sistema. Por eso con la globalización, se abrió paso a una discusión sobre las nuevas posibilidades en materia de autonomía (Drekonja, 2001; Russell \& Tokatlian, 2003; 2013).

Recientemente, una parte importante se ha dedicado a la gobernanza regional y a la integración, a propósito de la constelación de nuevas instituciones o bloques en la zona, como la Alianza Bolivariana para los Pueblos de Nuestra América (Alba), la Unión de Naciones Suramericanas (Unasur), la Comunidad de Estados Latinoamericanos y Caribeños (Celac) y la Alianza del Pacífico. A su vez existe un interés marcado por el fenómeno del giro a la izquierda desde finales de los noventa (Casta- 
ñeda, 2006, p. 28; L. Madrid, 2012; Cameron \& Hershberg, 2010).

Estas dos vertientes concentran una parte representativa de estudios sobre la política exterior pero ninguna se ha centrado en el caso ecuatoriano, pues el interés en los estudios por Estado, se orienta a casos que se consideren emblemáticos o representativos.

La literatura sobre las trasformaciones internas en Ecuador es abundante, lo cual se observa en trabajos sobre diseño institucional, política de coaliciones, politización de la justicia, neoconstitucionalismo y populismo (Mejía-Acosta \& Polga-Hecimovich, 2011; Pachano, 2010; Freidenberg, 2012). En lo referente a la política exterior ecuatoriana, aunque existan trabajos llamativos como los de Jeanne Hey (1995; 1993), Adrian Bonilla (2002; 2006), Javier Ponce Leiva (2006), Francisco Carrión Mena (2009) y Beatriz Zepeda (2009), tienen menos incidencia y visibilidad que los primeros.

\section{ECUADOR: CONSTRUCCIÓN DE ESTADO- NACIÓN Y POLÍTICA EXTERIOR}

El presente estudio propone analizar el rol del Ecuador en los diez años de Rafael Correa apoyándose en dos dimensiones. En primer lugar, en el proceso inacabado de construcción de Estado-nación (state-building) (Ayoob, 1995, pp. 4-12), que da cuenta de las dificultades internas, una vez instalada la democracia, y la forma como la vulnerabilidad externa afectó sus relaciones exteriores. El país experimentó una debilidad marcada frente a fenómenos externos (conflicto colombiano) y a una coyuntura en el sistema internacional (crisis financiera del su- deste asiático en 1997 y caída de los precios del petróleo). Segundo, y como consecuencia de esta condición que Ayoob denominaría tercermundista, Ecuador pasó por una refundación desde 2008 hasta 2011. Se trató de un cambio estructural y de identidad, no solo por aquello impuesto por el gobierno de Rafael Correa, sino porque la llamada "revolución ciudadana" incidió en todos los sectores del país, más allá de que hubiese sido positiva o negativa.

El método consiste en el análisis conceptual y del discurso apoyado en la revisión de fuentes secundarias y explorando el correlato entre dos variables. De un lado, la identidad ecuatoriana como la variable dependiente y los cambios internos provocados en el seno del proceso de construcción del Estado-nación como independiente. Este estudio parte de la premisa de separar la política internacional de la exterior, dos campos que suelen confundirse, pero cuya separación es fundamental, en especial en Estados periféricos que aparentemente tienen poca incidencia en el sistema internacional. De allí que no se apele a teorías en su conjunto para dar cuenta del caso en cuestión sino a conceptos específicos que, aunque no hagan parte de una sola teoría, se articulan alrededor del caso ecuatoriano.

El propósito consiste en retomar la idea fundacional de los estudios sobre política exterior de James Rosenau en la que se identifican tres estadios respecto de su diseño y ejecución. Se trata de una fase de iniciación que involucra todo tipo de gestiones emprendidas por actores nacionales para cambiar o modificar circunstancias de su entorno exterior. Luego de lo cual viene una fase de implementación y otra de reacción frente a hechos ocurridos en 
la política internacional que afecten o tengan incidencia en el Estado (Rosenau, 1968, pp. 311-312). En este caso se analiza la primera fase que consiste en aquella en la que se produce un reacomodamiento de fuerzas internas que pretenden cambiar el curso de la política exterior, pues se entiende que el ambiente afecta, de forma nociva, los intereses del Estado. En el caso de aquellos del tercer mundo es imprescindible observar el proceso desde adentro, sobre todo cuando ocurren tantos cambios en lapsos reducidos.

Este documento parte de la idea de Ecuador como un Estado periférico, debido a sus debilidades internas, su poca influencia en la esfera regional condición que podría considerarse incluso como aislamiento, sus niveles de desarrollo y su irrelevancia en el sistema internacional. Para John D. Martz, Ecuador es un Estado pequeño habida cuenta de su extensión territorial, su población, las limitaciones en materia de recursos y por la enorme influencia y capacidad que Estados Unidos ha ejercido, en especial para la explotación del petróleo que benefició a las empresas petroleras estadounidenses (Martz, 1996). En la misma línea, Adrián Bonilla tipifica la condición periférica ecuatoriana (Bonilla, 2002, p. 19) según los bajos niveles de institucionalización de las agencias públicas (incluyendo a los actores diplomáticos) y a la preeminencia del derecho para conducir su política exterior. En las propias palabras de Bonilla "los enfoques juridicistas dan cuenta de una difícil situación internacional caracterizada por la debilidad del Ecuador" (Bonilla, 2002, p. 19). Paralelamente, el autor ecuatoriano considera que se trata de un Estado pequeño por su ubicación en la zona andina convulsa y conflictiva, la "influencia estructural" de Washington y por sus relaciones con los vecinos (Bonilla, 2006, p. 166).

Bajo esta condición, e igualando la tendencia latinoamericana, el caso ecuatoriano fue estudiado bajo el prisma de la dicotomía independencia-autonomía. De allí que uno de los trabajos más referenciados sobre la política exterior ecuatoriana sea el de Jeanne Hey que aborda su dependencia aclarando que no se manifiesta de forma unívoca y más bien tiene múltiples facetas. La autora propone una clasificación de la dependencia en política exterior a partir de cuatro tipos: condescendencia, consenso, contra-dependencia y compensación (Hey, 1993). Las primeras describen una alineación con la potencia que puede ser por superioridad económica, y la segunda más por la vía de una negociación.

En este extracto se ve el papel que desempeña Estados Unidos en la idea de debilidad y de dependencia en Ecuador,

El resultado de una política exterior obediente de los latinoamericanos es claramente a favor de Estados Unidos. El proceso mediante el cual los líderes latinoamericanos han subyugado sus propias preferencias ha seguido por lo general tres caminos. Primero, de obediencia que responde a la presión abierta, a las amenazas, y a las promesas lanzadas desde el centro. Segundo, estas presiones pueden ser tácitas, es decir, Estados Unidos, no necesita amenazas expresas. Los líderes latinoamericanos son conscientes que pueden reaccionar a sanciones implícitas o potenciales o a estímulos respecto de Estados Unidos. Tercero, los tomadores de decisiones de la política exterior latinoamericanos siguen estos dictámenes en la medida en 
que la estructura del sistema internacional, tanto en su dimensión política como económica tiende a favorecer esas presiones desde el centro (Hey, 1993, pp. 546).

Otro trabajo de Hey evalúa los ciclos de política exterior ecuatoriana, desde el restablecimiento de la democracia hasta mediados de los noventa, justo antes de empezar la inestabilidad crónica. En el pasado reciente, el país transitaba en ciclos divididos entre el nacionalismo y la apertura. Un tímido nacionalismo presente en los gobiernos de Jaime Roldós Aguilera-Oswaldo Hurtado y Rodrigo Borja, y el neoliberalismo observado en los gobiernos de León Febres Cordero y Sixto Durán Ballén. En este texto aparece de nuevo la idea de alineación o emancipación respecto de Estados Unidos (Hey, 1995, p. 60).

En los períodos de acercamiento con Estados Unidos o de nacionalismo, las ideas imperantes eran aquellas de la élite, que podían o no reflejar el pensamiento de la sociedad. Comprobar el grado de sintonía entre las élites y la base social es una tarea relativamente simple. La alternación de gobiernos de una y otra corriente sugiere la necesidad de ir oxigenando el discurso exterior cada cuatro años, yendo de un lado al otro, sin que se impusiera en el largo plazo ni la autonomía ni la alineación. Andrew Yeo ha trabajado esta noción del "consenso de las élites" definido como una visión consistente de la política exterior, que además de ser el producto de un consenso entre los líderes, reflejaría creencias y valores compartidos sobre el ideal de la proyección (Yeo, 2010, p. 437).

No obstante, existe una idea por completar en los estudios de Hey sobre la política exterior ecuatoriana, pues todos parten del supuesto de que la dependencia y la emancipación se producen respecto de Estados Unidos (Hey, 1993, p. 546; 1995, p. 61). Sin embargo, la autonomía que se preconiza en los últimos años no solo tiene que ver con Washington, sino que debería tomarse en cuenta el pasado reciente con Perú y con Colombia para comprenderla.

De otra parte, se ha asumido que la construcción nacional o estatal se ha hecho o por la vía de la guerra como plantea Charles Tilly o por las rivalidades militares regionales como propone Miguel Ángel Centeno. Este último plantea una correlación entre la rivalidad militar (haya posibilidad real de guerra o no) y la capacidad de construcción del Estado (Centeno, 2002, p. 266). Fernando López-Alves añade a esta lógica la dimensión de la guerra antisubversiva como una variable a partir de la cual se reforzó la estatalidad (López-Alves, 2001, p. 164). En Ecuador, propiamente, sobresale el trabajo de Francisco Carrión Mena, para quien el conflicto con el Perú "estructuró la política exterior ecuatoriana hasta finales de los 90 y condicionó su inserción regional e internacional" (Carrión Mena, 2010, pp. 235-236). Por la forma como se finiquitó el tratado limítrofe, Ecuador quedó con la idea de que se le había impuesto una solución a la fuerza, quedando en evidencia su condición de debilidad. Se trata de una idea presente en los textos de algunos de los que participaron de dicha negociación como Enrique Ayala Mora y Carrión Mena quien la describe como "el triste epílogo de una confrontación militar que nunca debió producirse pero que culminó con la derrota del más débil: Ecuador" (Carrión Mena, 2009, p. 163). 
Este marco de análisis es aplicable parcialmente en Ecuador, pues si bien las rivalidades regionales con Perú fueron evidentes hasta los noventa, con Colombia la realidad es otra, pues se ha tratado de un sentimiento de vulnerabilidad, arraigado en la historia y confirmado por lo que Quito ha interpretado como agresiones constantes por parte de su vecino norte. Estas se pueden evidenciar en las fumigaciones en la zona fronteriza, las acusaciones y exigencias para que Ecuador tomara partido en el conflicto colombiano. Quito se terminó alejando de su tradición en el gobierno de Lucio Gutiérrez cuando se intentó mediar en el conflicto colombiano (Ponce Leiva, 2006, p. 154) sin lograr efectos positivos y lo que fue peor para Quito: derivó en la actitud hostil del entonces gobierno de Álvaro Uribe Vélez. Es decir, entre Colombia y Ecuador no ha existido una rivalidad militar, sino el sentimiento de vulnerabilidad del segundo.

Descartando la rivalidad militar como el principal elemento de estatalidad ecuatoriana, una de las tesis de las que parte el presente texto es que la principal variable de construcción estatal correspondió a la búsqueda de una modernidad inacabada, para lo cual se apeló: a) al desarrollismo bajo la idea del modelo cepalino; b) al populismo en especial en los gobiernos de José María Velasco Ibarra; y c) al discurso burocrático nacionalista implantado por los militares. Se observa, por tanto, que las rivalidades militares o territoriales regionales fueron solo elementos secundarios.

En cuanto a la política exterior, tras la democratización en 1979, Ecuador tuvo tres períodos representativos. Primero tras la instalación de administraciones civiles, se vivió una proyección cíclica, marcada por acercamientos y distanciamientos con EE.UU., como ya se mencionó.

Segundo, el levantamiento indígena de 1990, sumado al papel protagónico de estos en la salida de Jamil Mahuad a finales de los noventa, mostró la trasformación de la sociedad ecuatoriana, que se sentía cada vez menos representada por los partidos políticos. Las protestas en 1990 y 1999 no solo fueron protagonizadas por indígenas, sino por varios sectores sociales e independientes, que hacían pensar en grandes pasivos del proceso democratizador de finales de los 70. En 1997 con la destitución de Abdalá Bucaram y el período de convulsión subsiguiente, el país sufrió un ensimismamiento que lo alejó de la escena internacional. El aspecto más sobresaliente consistía en su debilidad y vulnerabilidad frente a Estados Unidos. Aprovechando uno de los peores momentos en la historia del Ecuador en 1999, el gobierno de Bill Clinton propuso a su homólogo Jamil Mahuad, la instalación de una base militar para la interdicción aérea en la ciudad costera de Manta. Esto convertía al país en un aliado estratégico para la lucha contra el narcotráfico, pero con efectos en la soberanía. Años más tarde, en el Plan de Política Exterior hacia 2020 (Planex, 2020) quedaría consignada la voluntad ecuatoriana de no volver a permitir la instalación de bases foráneas. Distintos sectores de la sociedad ecuatoriana dejaron claro su rechazo a tales concesiones. La crisis financiera de 1999, la peor en toda la historia del país que terminó en el llamado feriado bancario (congelación de los activos en la banca), puso de manifiesto la marcada vulnerabilidad externa. Las tres causas 
del colapso tuvieron que ver con fenómenos de afuera: el estallido de la burbuja inmobiliaria en el sudeste asiático que produjo una crisis de confianza en los inversores; la caída de los precios del barril del petróleo; y las inundaciones por el fenómeno del niño a finales de ese año (Jaramillo Jassir, 2007).

Y, tercero, con la "revolución ciudadana", Ecuador buscó un renacimiento exterior, aprovechando los niveles de cohesión interna, que para muchos solo se explican por el autoritarismo. De esta forma, pasó de ser un Estado irrelevante en la zona, a un líder en ciernes que, aunque lejos de volverse suficientemente influyente, ganó visibilidad en determinados temas.

\section{ECUADOR ENTRE 2007 Y 2017: ¿CÓMO ENTENDER SU CAMBIO EN POLÍTICA EXTERIOR?}

Desde que inició el gobierno de Correa, la reforma del sistema político y económico fue notoria. La trasformación se cristalizó en la Constitución de 2008 que sintetizó dos procesos que en el pasado se entendían como secuenciales: la construcción de Estado-nación en primera instancia y la posterior consolidación democrática. Sin embargo, puede ocurrir, como en el caso de los neoconstitucionalismos de la región andina, que a la par de la refundación estatal se consolide la democracia. Esto se percibió en 1999 en Venezuela, en 2008 en Ecuador y en 2009 en Bolivia. Tres Estados que antes habían sufrido severas crisis políticas, pero que por reformas en sus cartas magnas reafirmaron el papel del Estado y paralelamente hacían un intento por profundizar la democracia (Andrade y Olano, 2005; De la Torre y Peruzzotti, 2008; Jaramillo Jassir, 2011).

La transformación en política exterior en el gobierno de Correa fue apenas estudiada. Sobresale en este aspecto, el artículo de Beatriz Zepeda y María Gabriela Egas sobre la política exterior de la "revolución ciudadana". A pesar de constituir un análisis detallado de la evolución desde la finalización del litigio con el Perú y la entrada de Ecuador en el círculo del conflicto colombiano, el estudio se centra en la propuesta diplomática de Correa, más que en su política exterior. La diplomacia está ligada a los canales formales de relacionamiento entre los Estados y deja por fuera una serie de interacciones cada vez más relevantes ${ }^{3}$ (Zepeda \& Egas, 2011, p. 101).

También cabe destacar el trabajo de Carlos Malamud y Carola García de 2009 bajo la etiqueta "La política exterior de Ecuador: entre los intereses presidenciales y la ideología” (Malamud \& García-Calvo, 2009). Es un buen insumo para el análisis del rumbo exterior de ese gobierno, pero se trata de un análisis coyuntural, que cae en el vicio de adjudicar todas las orientaciones al personalismo y al presidencialismo. Se trata de un rasgo que, aunque evidente, está sobrevalorado y desconoce dinámicas con las que se fue construyen-

\footnotetext{
3 La política exterior consiste en el conjunto de acciones de un actor internacional hacia el sistema, y no se limita a los actores formales, sino que incluye a ministerios y agencias diferentes del Ministerio de Relaciones Exteriores, así como actores no estatales.
}

OASIS, ISSN: 1657-7558, E-ISSN: 2346-2132, N³2, Julio- Diciembre de 2020, pp. 153-175 
do una nueva identidad no solo adjudicable al estilo de Correa. El escrito concluye con la idea suficientemente expandida, pero sin justificación plena, de que Correa debía moverse entre dos macro-proyectos regionales, el de Brasil liderado por Luiz Inácio Lula da Silva, y el venezolano chavista.

La primera de las conclusiones del análisis va en esa dirección:

El carácter personalista que imprime Correa a la política exterior ha conducido a la diplomacia ecuatoriana a moverse entre la adhesión al proyecto bolivariano de Hugo Chávez y su cercanía al más moderado Lula da Silva. De momento, Correa no termina de definirse al respecto, especialmente por el incumplimiento de muchas de las promesas venezolanas. Por eso, no ha concretado su ingreso al Alba y prefiere mantenerse como observador y no como miembro pleno de la organización bolivariana. En este difícil equilibrio, el acercamiento a Chávez ha significado en términos regionales la prolongación del enfrentamiento con Colombia y la apertura de una crisis que afecta la lucha contra el tráfico de drogas y antiterrorista (Malamud \& García-Calvo, 2009, p. 7).

Tampoco es cierto que la principal característica sea el personalismo, pues precisamente uno de los temas donde se otorgó mayor autonomía ministerial fue en relaciones exteriores donde aparecieron figuras como María Fernanda Espinosa, Fander Falconi, Ricardo Patiño y Guillaume Long. Este último tal vez sobre el que Correa tenía mayor ascendencia, pero aun así se trató de perfiles con amplio margen de maniobra. La búsqueda de mayor presencia en la institucionalidad regional (CAN, Unasur, Celac y Otca ${ }^{4}$ ) obligó a un esfuerzo por delegar en varias dependencias del Ministerio, asuntos que se fueron diversificado y desarrollando cada vez más.

Ambos artículos suponen contribuciones a la comprensión de las acciones externas del Ecuador bajo Rafael Correa, pero ninguno aborda la transformación de la identidad.

\section{EL PERFIL DE CORREA Y SU LECTURA REGIONAL ANTES DE SER PRESIDENTE}

Una característica importante del proceso ecuatoriano es la relevancia de la política exterior para Rafael Correa incluso antes de ser presidente. Su carrera hacia la presidencia empezó con temas regionales controvertidos, en especial por su amistad con Hugo Chávez.

Luego de la caída del gobierno de Lucio Gutiérrez y con llegada de Alfredo Palacio, Correa asumió como ministro de economía en abril de 2005. Sus diferencias con el presidente fueron inocultables a raíz del tratado de libre comercio con Estados Unidos, pues Correa consideraba que solo debía incorporarse previa consulta popular. También se presentaban controversias respecto de la postulación de Luis Alberto Moreno como secretario general del Banco Interamericano de Desarrollo (BID) a la que se oponía Correa, ya que este había efectuado toda la labor de cabildeo durante el

4 Organización del Tratado de Cooperación Amazónica. 
gobierno de Andrés Pastrana, para la obtención de recursos del Plan Colombia, que tanto daño había causado al Ecuador a juicio del entonces ministro (Lukas, 2007, p. 41).

Finalmente, el tema del petróleo fue el más determinante en la carrera política de Correa, ya que le sirvió de plataforma para su posterior campaña presidencial. El entonces ministro de economía reformó el Fondo de Estabilización, Inversión y Reducción del Endeudamiento Público (Feirep) para destinar menos recursos a la deuda y más a la inversión social. Un 40\% de los mismos se orientarían a la reactivación productiva y a la creación de empleo, un $10 \%$ para ciencia, tecnología y fortalecimiento del capital humano, y $15 \%$ como máximo para el pago de la deuda externa ( $E l$ Universo, 2005).

Con esto se observa una posición clara respecto de la política exterior ecuatoriana, y una fuerte presunción de que se habían cometido errores, que urgía corregir desde el modelo. Aquello condujo a la inevitable renuncia, en agosto de 2005, a través de una carta difundida en medios y que contribuyó a su popularidad.

La campaña presidencial enfrentó a dos modelos político-económicos irreconciliables. De un lado, una óptica ultraliberal con las candidaturas de Álvaro Noboa y Cynthia Viteri, que le apuntaba a una apertura económica, jalonada por la empresa privada, con la idea de corregir algunos elementos del modelo existente. De otro, la propuesta de Correa y de León Roldós que criticaba la excesiva liberalización de la economía y proponía una participación más activa del Estado en la redistribución y en las reglas de juego del sistema financiero (Paz y Miño Cepeda, 2006, p. 2).
Se presentaron, además, candidatos sin mayores posibilidades de llegar a la presidencia como en el caso del líder indigenista Luis Macas, Luis Villacís de la izquierda radical, y Gilmar Gutiérrez del Partido Sociedad Patriótica y hermano del depuesto presidente, entre otros.

El 15 de octubre se daba a conocer que Noboa y Correa llegaban a la segunda vuelta con el $27 \%$ y $22 \%$ respectivamente (El Universo, 2006). Esto hacía presagiar una campaña para la segunda vuelta polarizada entre los dos modelos, en la que Correa se terminó imponiendo.

Rafael Correa, llegó con una apuesta con miras a la segunda vuelta bastante arriesgada, pues desistió de proponer listas al Congreso, en consonancia con el proyecto de una nueva constitución. El enfrentamiento derivó en la conocida victoria de Correa, que reflejó el deseo de un porcentaje representativo de ecuatorianos por un cambio estructural. La victoria del candidato de Alianza PAís más que corresponder a una inclinación del electorado hacia la izquierda, se explicó por un reclamo de cambio, anclada en una crítica vehemente a los partidos tradicionales, y al poder omnímodo del que gozaban, en lo que Correa denominó "partidocracia".

\section{LA DIMENSIÓN EXTERIOR DE LA "REVOLUCIÓN CIUDADANA"}

Durante los diez años del gobierno de Correa se reivindicó intensamente la idea de una ruptura ¿qué tan válida es plantearlo en política exterior? Y más relevante aún ¿en qué aspectos se puede apreciar tal rompimiento? La respuesta respecto a la primera pregunta es 
ambigua, pues Correa parece haber retomado una tradición de la diplomacia ecuatoriana vigente durante el gobierno militar de Guillermo Rodríguez Lara (1972-1975) y en las primeras administraciones de la era democrática. En los años del citado general se tomó distancia respecto de Washington, Ecuador ingresó al G77 en Naciones Unidas y se convirtió en observador del Movimiento de Países No Alineados (Ponce Leiva, 2006, p. 139). Un hecho significativo del acento autónomo de la política exterior durante y posterior a la transición consistió en la ruptura de relaciones diplomáticas con el gobierno de Anastasio Somoza en el último gobierno militar del general Alfredo Poveda. En consecuencia, Jaime Roldós, quien inauguró la era civil, se convirtió en el primer presidente de América Latina en visitar a Nicaragua una vez consumado el triunfo de esa revolución (Avilés Zambrano).

La trasformación en el marco de la "revolución ciudadana" derivó en un régimen que tomó distancia de lógicas del mundo occidental, claramente racional y liberal. La inclusión de una concepción de la política, inspirada en principios quechuas como el sumak kawsay o buen vivir es una muestra. La manera en que Ecuador trató de reivindicar frente a Colombia una serie de abusos cometidos por el segundo evidenciaba el reflejo del mencionado principio. "Ecuador ama la vida" se convirtió en la divisa a partir de la cual se trabajaron tres frentes en materia de política exterior.
El primero orientado a las denuncias de la aspersión aérea de glifosato ordenadas por el gobierno colombiano a finales de 2006 y comienzos de 2007. Bogotá se había comprometido a no fumigar en una franja de 10 kilómetros desde la frontera, pero la administración de Álvaro Uribe Vélez decidió retomar las aspersiones sin consultar a su vecino.

Cuando se rompieron relaciones en 2008, a raíz del ataque colombiano a Santa Rosa de Sucumbíos, el gobierno ecuatoriano decidió, en un hecho histórico, demandar a Colombia ante la Corte Internacional de Justicia. Y por primera vez en mucho tiempo, Quito se impuso sobre Bogotá, en una conciliación por anticipado en la que Bogotá prefirió compensar con 15 millones de dólares a su vecino, antes de arriesgarse a una condena mayor en dicho tribunal (El Telégrafo, 2013). Esto cambió la percepción del país en la zona, pues transitó de una historia de extrema vulnerabilidad con sus vecinos, marcada por un mal arreglo fronterizo con el Perú (Ardila, 2003, p. 235) y las agresiones constantes de Colombia en el marco de la guerra contra las guerrillas, a obtener una compensación pecuniaria y política. Ecuador recreó una identidad como un actor capaz de combatir las hegemonías regionales usando como herramienta el derecho, y más allá de lo jurídico, se debe reconocer que alcanzó a establecerse como un defensor del medio ambiente. Su idea de imponer una imagen como Estado que "amaba la vida", estuvo siempre ligada precisamente a su condición amazóni-

5 Este grupo, creado en 1964, ha buscado la vocería de los países en vías de desarrollo dentro de la organización. 
ca y el haber sufrido por cuenta de su vecino del norte.

El segundo fue la iniciativa del Yasuní ITT, que consistía en la protección de una reserva en la Amazonía, con un comprobado potencial de yacimientos en petróleo y que se comprometió a no explotar a cambio de recursos por parte de la comunidad internacional, en compensación por su preservación. Ecuador debía recibir 3600 millones de dólares, por cuenta de la iniciativa que podía servir de modelo en otras latitudes. No obstante, el gobierno sorprendió al anunciar en 2013, que modificaría el plan inicial y que una parte de la reserva sería explotada (Jaramillo Jassir, 2013). Esto se justificó en que no se había recibido el monto pretendido y aclarando que no se alteraría el equilibrio ambiental de la zona. Obviamente, el anuncio cayó muy mal y se interpretó como un gesto demagógico y como una clara inclinación hacia el tema económico en detrimento del ambiental. El gobierno insistió en que la reserva, ubicada en el Amazonas, estaba en medio de una de las regiones más pobres y que urgía la extracción de algunos de sus recursos.

Esto produjo un intenso debate en el interior del país, con pocas connotaciones internacionales, aunque paradójicamente el país mantuvo la imagen de compromiso con el ambiente. Ecuador tuvo una activa participación en iniciativas como la Conferencia entre Partes (COP21) de 2015 en la que mostró un compromiso por reivindicar el ambiente como derrotero de su política exterior. En el caso ecuatoriano, esto va más allá de la simple retórica, pues lo ocurrido en los últimos años por cuenta de catástrofes naturales, así como la expansión del conflicto colombiano, han hecho evidente su vulnerabilidad en materia ambiental.

Por oposición a Colombia, Quito optó por una narrativa de paz redituable en espacios internacionales. Correa fustigó al Plan Colombia al que consideraba guerrerista -incluso antes de ser presidente como se describió- (Cramer, 2007). Por eso propuso el Plan Ecuador, acudiendo de nuevo al antagonismo con el vecino que, en ese entonces, era asociado con el militarismo y se le etiquetaba como el Israel de América Latina, como alguna vez sostuvo Hugo Chávez. En 2010, el recién posesionado Juan Manuel Santos dijo sentirse orgulloso de que Colombia portara esa denominación (Comunicas, 2010), una señal clara de dos identidades en constante oposición y que favorecieron la idea de un Ecuador pacifista.

Y, sin duda, la inclusión de la movilidad humana fue una de las transformaciones más llamativas. El Ministerio no solo se denominó de Relaciones Exteriores, sino que se añadió la movilidad humana a la cual se dedicó un Viceministerio. El derecho a la movilidad que había sido elevado al estatus constitucional por el texto de Montecristi ${ }^{6}$ de 2009 se convirtió en un elemento constitutivo del discurso correísta hacia el exterior. Después se aprobó la Ley Orgánica de Movilidad convirtiendo al país en pionero en la materia. Con esto Correa

6 Lugar donde se redactó la constitución de 2009, por eso algunos la denominan de esa forma. 
entendía reconciliarse con la tradición diplomática, pues el país tenía un largo recorrido de normas favorables a la migración como la Ley de Extranjería de 1867 o al Decreto del 25 de noviembre de 1867 que le otorgaba la ciudadanía a las personas que llegaban de Chile, Colombia, Perú y Venezuela (Ramírez, 2013, p. 15). Aunque Ecuador pretendía convertirse en un referente de movilidad en tanto que garantía humana y no como una concesión de los Estados, dicha política tuvo que reconducirse, pues al eliminar el sistema de visados se produjeron varios problemas por la llegada masiva de migrantes cubanos y haitianos. De esta manera, el gobierno impuso de nuevo restricciones migratorias para algunas nacionalidades.

Esto puso en evidencia las principales dificultades que experimentó Correa durante sus diez años, para producir algunos cambios en la proyección exterior. Aunque algunas iniciativas revolucionarias pretendían generar cambios inmediatos, estos se vieron truncados por la propia realidad y por dinámicas que terminaron condicionando la puesta en marcha de iniciativas emblemáticas como sucedió con la migración.

\section{CONCLUSIONES: ¿CÓMO INTERPRETAR EL CAMBIO DE IDENTIDAD EN ECUADOR?}

La identidad se entiende como el conjunto de rasgos y características que definen y diferencian a un Estado en el sistema internacional. La misma es el resultado de la proyección externa a través de actos discursivos y de su contextualización social. El constructivismo sirve para entender que el rol de Ecuador en el plano internacional no puede explicarse solo por sus recursos, ni su posición estructural, pues si por ello fuera, sería un Estado irrelevante y sin posibilidades de proyección.

Por ello, es indispensable traer a colación los cuatro aportes del constructivismo a la política exterior, según Arlene Tickner quien los emplea para el caso del cambio de identidad en Colombia (Tickner, 2002, pp. 370-371). Según la autora, se parte del supuesto de que los Estados participan en "la construcción de sus respectivos mundos”, y en política exterior, estos agentes escogen aquello que "interpretan, deciden, pronuncian e implementan" (Smith, 2001, p. 38). Las identidades, por tanto, son el resultado de la interacción social con otros, principio clave en la identidad ecuatoriana, especialmente en su relación con Colombia, Estados Unidos, Venezuela o Perú. En complemento se afirma que la identidad se constituye de la alteridad o de la diferenciación con respecto de otros.

¿Cuándo y cómo cambió la identidad ecuatoriana? La transformación de la identidad ecuatoriana no se explica exclusivamente por la recuperación económica, sino por el cambio discursivo y la autopercepción. Tampoco es cierto que toda la mutación se deba a una decisión emprendida por el gobierno de Correa, ni por el cambio en su discurso, sino que tiene que ver la intersubjetividad (Wendt, 1999, p. 160) surgida por la forma en que Ecuador construyó una narrativa y el resto de naciones de la región la asimiló.

Los actores internacionales se dotan de una identidad, relativamente estable, que comporta un rol determinado y una expectativa sobre sí mismos, constituyendo significantes 
colectivos (Wendt, 1992, pp. 398-399). La identidad que se presenta bajo cuatro tipos (personal, de tipo, de rol o colectiva) (Wendt, 1999, p. 224) en Ecuador es claramente personal, pues lo desmarca de otros y le significa un sello particular. El interés nacional que se construyó discursivamente desde comienzos de 2007 fue el resultado de un proceso histórico en el que no solo tuvo que ver Rafael Correa, sino el conjunto de hechos que fueron cambiando la política ecuatoriana. Se trata de la transformación del país en la década de los noventa por cuenta de la prolongada crisis y el proceso refundacional posterior. La "revolución ciudadana", antecedida por hechos determinantes es, ante todo, una transformación del significado que Ecuador tiene de sí mismo y en la región.

Lo anterior se puede apreciar en la soberanía, principio constitutivo del sistema internacional que no puede ser asumido en un sentido exclusivo legalista-político, sino como un término polisémico. Para Correa la soberanía se convirtió en un rasgo fundamental de la política exterior tratando de tomar distancia de Estados Unidos y de Colombia, apelando a la no injerencia para rechazar el militarismo de ambos. Igualmente, promovió la defensa del medioambiente criticando las relaciones de producción en el mundo, como reflejo de su lectura marxista y progresista. Bajo esta lógica, se embarcó en la defensa de Julián Assange, convirtiéndola en una pugna cuyo trasfondo era el centro y la periferia.

El constructivismo es enfático al señalar que las fuentes de la identidad nacional no solo se agotan en el espectro interno, sino que están condicionadas por los cambios que ocurren en el sistema internacional o la percepción de terceros Estados. Para el caso ecuatoriano, se parte de la premisa de que lo que Martin Marcussen bautizó como "coyunturas críticas" (critical junctures), pueden generar y recrear la identidad. Se trata de "situaciones percibidas como crisis, resultado de fracasos políticos completos, pero igualmente generados por hechos externos" (Marcussen, 2001).

El resultado más evidente de esa identidad fue el liderazgo suramericano, jamás alcanzado en el pasado. En diciembre de 2014, se inauguró la Sede de la Secretaría General de la Unasur, en la Mitad del Mundo en San Antonio de Pichincha. Un lugar cargado de simbolismo porque rescataba al país como centro del mundo y desde esa posición pretendía convertirse en referente de la unidad regional. Sin duda alguna, la principal apuesta en materia de política exterior para el país fue Unasur, lo cual se observó no solo en la propuesta de Correa de convertirse en anfitrión de esa Secretaría, sino en el impulso que su cancillería le otorgó al bloque en proyectos concretos como el de una ciudadanía regional, la infraestructura regional, y la creación de la Escuela Suramericana de Defensa (Esude) que tendría como sede también a la Mitad del Mundo?.

\footnotetext{
7 El proyecto quedó congelado por la crisis posterior que vivió Unasur y por el anuncio de Lenin Moreno de destinar la sede de la Secretaría General a una universidad para los pueblos indígenas.
} 
2015 fue un punto de inflexión en la política exterior y Ecuador consolidó una serie de reformas internas que cumplieron una fase amplia desde la disolución del Congreso, pasando por la Constitución de Montecristi, hasta la reforma de 2011 por medio de una consulta popular. Desde el discurso oficial se aseveraba constantemente que algunas de esas reformas le devolvieron al país la autoestima y le dotaron de un rol (Long, 2016).

En la última etapa del período de Correa, Ecuador buscó una participación activa en tres temas vitales para la zona suramericana: la crisis prolongada entre Colombia y Venezuela, los diálogos de paz entre el gobierno colombiano y el Ejército de Liberación Nacional (ELN) y la búsqueda constante porque Unasur no perdiera protagonismo, pues desde ese entonces, las rivalidades entre algunos Estados auguraban crisis futuras.

Para explorar el cambio de la política exterior ecuatoriana, es fundamental entender las condiciones en las que se fue trasformando su identidad y que se pueden resumir en los siguientes hechos que significaron un punto de inflexión:

- La firma de la paz con el Perú en 1998, justo en momentos en que la región denunciaba los efectos nocivos del Plan Colombia y la regionalización del conflicto colombiano.

- La actitud hostil de Colombia que provocó un profundo malestar a partir del cual se recreó un sentimiento de unidad nacional, reafirmando el carácter pacífico que por siglos ha marcado el talante ecuatoriano. Es de notar que, en todo el período de crisis desde mediados de los noventa hasta comienzos de siglo, no hubo episodios serios de violencia política. Existe claro está, la excepción de los movimientos armados Alfaro Vive ¡Carajo! y Montoneros Patria Libre que duraron muy poco y cuya violencia jamás alcanzó las dimensiones de otras guerrillas urbanas o rurales en América Latina.

- La llegada al poder de un apolítico como Rafael Correa que reivindicaba una independencia de la política tradicional prometía revivir los valores de la revolución alfarista de finales del siglo xix que significó la liberalización más importante en su historia. Esa reconexión con la génesis de la modernidad ecuatoriana fue vital para la regeneración de una identidad.

- El otorgamiento de un estatus constitucional a valores indígenas y ancestrales como el Buen Vivir (sumak kawsay) cambió la autopercepción y la de terceros sobre Ecuador, dotándolo de un rol que no había tenido en el pasado.

Una de las vetas que se abre después de revisar este caso, tiene que ver con la forma en que la política exterior va reflejando el cambio o la evolución del sistema político. En Ecuador, resulta llamativo que converjan tres procesos: la puesta en marcha del restablecimiento del Estado, la consolidación democrática y un despertar exterior.

Esta lectura se articula con algunas de las nociones que abogan por entender la política exterior de los Estados periféricos, pequeños o del tercer mundo, observando variables internas y no solo el proceso de toma de decisiones, 
en el que se suele ver tan solo una parte, pero difícilmente se pueden observar alteraciones en la identidad. Estos cambios merecen la puesta en perspectiva de tres tipos de teorías: las relativas a la democratización (una etiqueta que normalmente depende del contexto histórico y del autor), al Estado y concretamente al proceso inacabado en países del tercer mundo como los denomina Ayoob, y a aquellas que insisten en entender la política exterior no solo como un proceso racional, sino como el punto de llegada de narrativas encontradas en el marco de la intersubjetividad.

En el caso ecuatoriano se pudo comprobar una alteración de la identidad empujada por el proceso de democratización que, de todos modos, fue imperfecto durante los diez años de Rafael Correa, pues se produjeron algunas manifestaciones de autoritarismo que retrasaron ese cambio de identidad deliberado. Es decir, el cambio de la identidad tiene dos dimensiones, una deliberada que autores como Wendt denominan de roly otra que se produce por la interacción de subjetividades cuando un Estado va cambiando desde adentro, pero la percepción exterior puede diferir de los propósitos y la transformación identitaria termina escapando al control de los gobiernos. Ningún Estado está en la capacidad de controlar o manejar en términos absolutos su identidad, pues resulta inviable manejar o controlar la percepción de terceros en un mundo donde abundan las informaciones en tiempo real y el control de los medios es imposible, incluso en Estados de corte autoritario.

Al observar los cambios en la política exterior ecuatoriana se tiende a pensar que todo obedece a su carácter presidencial tal como lo plantea Adrián Bonilla quien considera que sus características "se subordinan a las del orden político [...] un régimen presidencialista fundamentado en relaciones [...] de dominación que se expresan en patrimonialismo, clientelismo o liderazgo caudillista" (Bonilla, 2006, p. 166). Tal aseveración sugiere una política exterior extremadamente dependiente de una sola persona que concentra el poder presidencial. No obstante, en este artículo se pudo observar la forma como el giro identitario en Ecuador obedece a un proceso más complejo en el que no solo converge el liderazgo caudillista o populista de un líder, sino de movimientos sociales, contradicciones históricas propias de la democratización imperfecta (incluso fallida), circunstancias externas como el fenómeno migratorio, el proceso de integración cambiante en la región e incluso la emancipación respecto de Estados Unidos, favorecida por ese progresismo que algunos años atrás era hegemónico en la región, situación que fue capitalizada por Correa.

Esta nueva lectura tiene como deber inaplazable, el superar el debate abstracto sobre el vínculo interno-externo, y empezar a asociar la política exterior con conceptos muy presentes en los Estados de la periferia como la democratización imperfecta, el populismo, las representaciones de los litigios o conflictos vecinales, y los imaginarios sobre el Estado ideal.

\section{REFERENCIAS}

Acharya, A. (2014). Rethinking Power, Institutions and Ideas in World Politics: Whose IR? Nueva York: Routlegde. 
Allison, G. y Zelikow, P. (1999). Essence of Decision: Explaining the Cuban Missile Crisis. New York: Longman.

Andrade, P. y Olano, A. (2005). Constitucionalismo autoritario: los regímenes contemporáneos en la región andina. Quito: Universidad Andina Simón Bolívar.

Ardila, M. (2003). Viabilidad de la seguridad regional en el área andina. En W. Grabendorff, La seguridad regional en las Américas, (pp. 225-251). Bogotá: Fescol.

Aviles Zambrano, F. (18/07/2019). La huella de la revolución sandinista en Ecuador. El Universo.

Ayoob, M. (2002). Inequality and Theorizing in International Relations: The Case for Subaltern Realism. International Studies Review 4, no 3 (otoño): 27-48.

Ayoob, M. (1995). The Third World Security Predicament. State making, regional conflict, and the International System. Londres: Lynne Rienner.

Beck, S. y Mijeski, K. (09/2001). Barricades and Ballots: Ecuador's Indians and the Pachakutik Political Movement. Estudios Ecuatorianos, $\mathrm{n}^{\circ} 1$.

Bernal, J. L. (2015). Colombia e Israel bajo la administración Uribe: compañeros en la guerra global contra el terrorismo. Colombia Internacional, 71-106.

Bonilla, A. (2006). Política exterior del Ecuador: 25 años de vulnerabilidad. Revista AFESE, No. 44: 165-181.

Bonilla, A. (2002). Alcances de la autonomía y hegemonía de la política exterior ecuatoriana. En Bonilla, A. (editor), Orfeo en el infierno. Una agenda de la politica exterior ecuatoriana (pp. 11-45). Quito: Flacso.

Cameron, M. y Hershberg, E. (2010). Latin America's Left Turn. Politics, policies, trajectories of change. Colorado: Lynne Rienner.

Castañeda, J. (2006). Latin America's Left Turn. Foreign Affairs.
Carrión Mena, F. (2009). La paz por dentro: EcuadorPerú, testimonio de una negociación. En Revista AFESE, No. 50, 163-169.

Carrión Mena, F. (2010). El conflicto limítrofe con Perú como eje ordenador de la política exterior ecuatoriana. En Zepeda, B. (comp.), Ecuador: relaciones internacionales a la luz del bicentenario (pp. 233- 264). Quito: Flacso.

Centeno, M. A. (2002). Blood and Debt: War and the Nation-State in Latin America. Pennsylvania: The Pennsylvania State University Press.

Comunicas. (07/06/2010). Santos se siente "muy orgulloso" de que se compare Colombia con Israel. Comunicas.

Cramer, D. (26/04/2007). Correa lanza el Plan Ecuador para contrarrestar el Plan Colombia. Página 12.

De la Torre, C. y Peruzzotti, P. (2008). El retorno del pueblo. Populismo y nuevas democracias en América Latina. Quito: Flacso.

Deutsch, K. (1957). Political Community in the North Atlantic Area: International Organization in the Light of Historical Experience. Princeton: Princeton University Press.

Drekonja, G. (01/04/2001). Nuevos retos de la política exterior latinoamericana frente al siglo xxi. Colombia Internacional, $\mathrm{n}^{\circ}$ 51, 51-66.

El Telégrafo. (14/09/2013). Con \$ 15 millones, Colombia compensará por fumigaciones. El Telégrafo.

El Universo. (19/05/2005). Ministro de economía explica plan de reformas del Feirep al Congreso. El Universo.

El Universo. (15/10/2006). Noboa y Correa a segunda vuelta según resultados inmediatos de E-Vote. El Universo.

El Universo. (05/08/2005). Rafael Correa renunció al Ministerio de Economía. El Universo. 
Fearon, J. (1998). Domiest Politcs, Foreign Policy, and Theories of International Relations. Annual Review of Political Science, 289-313.

Finnemore, M. (1996). National Interests in International Society. Londres: Cornell University Press.

Freidenberg, F. (2012). Ecuador 2011: Revolución ciudadana, estabilidad presidencial y personalismo político. Revista de Ciencia Politica 32, n 1, 129-150.

Giacalone, R. (2012). Latin American Foreign Policy Analysis: External Influences and Internal Circumstances. Foreign Policy Analysis, 335-354.

Haas, E. (1958). The Uniting of Europe: Political, Social, and Economic Forces 1950-1957. California: Stanford University Press.

Hey, J.A.K. (1995). Ecuadoran Foreign Policy Since 1979: Ideological Cycles or a Trend towards Neoliberalism? Journal of Interamerican Studies and World Affairs, 37(4), 57-88.

Hey, J.A.K. (10/1993). Foreign Policy Options under Dependence: A Theoretical Evaluation with Evidence from Ecuador. Journal of Latin American Studies, 543-574.

Hill, C. (2003). Tha Changing Politics of Foreign Policy. Nueva York: Palgrave.

Jaramillo Jassir, M. (2007). Aplicación de conceptos para el estudio de la inestabilidad política como amenaza a la seguridad de las naciones andinas: El caso ecuatoriano. Papel Politico, 565-590.

Jaramillo Jassir, M. (20/08/2013). ¿Por qué abandonar Yasuní?, El Espectador.

Jaramillo Jassir, M. (2011). La desilusión democrática en la Región Andina. Criterio Libre, 307-328.

Katzenstein, P. (1976). International Relations and Domestic Structures: Foreign Economic Policies of Advanced Industrial States. International Organization, Invierno, 1-45.
Keohane, R. (1969). Review: Liliputians' Dilemmas: Small States in International Politics. International Organization, 291-310.

Keohane, R. y Nye, J. (2001). Power and Interdependence. Nueva York: Longman.

Kowert, P. (1998). Agent versus Structure in the Construction of National Identity. En Kowert, P.; Kubalkova, V. y Onuf, N., International Relations in a Constructed World (pp. 101-123). Nueva York : M. E. Sharpe.

Krasner, S. (1977). United States Commercial and Monetary Policy: Unravelling the Paradox of External Strengh and Internal Weakness. International Organization 31, no 4 (Otoño), 635-671.

L. Madrid, R. (2012). The Rise of Ethnic Politics in Latin America. Cambridge: University Press.

Long, G. (12/05/2016). Conferencia del Ministro de Relaciones Exteriores de Ecuador en la Universidad del Rosario. Bogotá.

López-Alves, F. (2001). The Transatlantic Bridge: Mirrors, Charles Tilly, and State Formation in the River Plate. En Centeno, M.A., The Other Mirror: Grand Theory through the Lens of Latin America (pp. 153-176). Princeton: Princeton University Press.

Lukas, K. (2007). Rafael Correa: un extraño en Carondelet. Quito: Planeta.

MacLeod, A. (2002). L'approche constructuviste de a politique étrangère. En Charilon, F. Politique Étrangère. Nouveaux regards (pp. 65-89). París: Presses de Sciences Po.

Madrid, R. L. (2008). The Rise of Ethnopopulism. World Politics, 475-508.

Malamud, C. y García-Calvo, C. (04/2009). La política exterior de Ecuador: entre los intereses presidenciales y la ideología. Análisis del Real Instituto Elcano (Real Instituto Elcano), no 61. 
Marcussen, M. (2001). Constructing Europe? The Evolution of Nation-State Indentities. En Christiansen, T.; Jorgensen, K.E. y Wiener, A., The Social Construction of Europe, Cambridge: University Press.

Martz, J. (s.f.) The Fate of a Small State. Ecuador in Foreign Affairs. Latin American Nations in World Politics, Nueva York: Routledge.

Massal, J. (01-06/2010). El proyecto político indígena ecuatoriano convergencia y divergencias con su entorno político. Colombia Internacional, $\mathrm{n}^{\mathrm{o}}$ 71, 9-33.

Mejía-Acosta, A. y Polga-Hecimovich, J. (2011). Coalition Erosion and Presidential Instability in Ecuador. Latin American Politics and Society 53, $\mathrm{n}^{\circ} 2$ (primavera): 87-111.

Moravcisk, A. (1993). Introduction: Integrating International and Domestic Theories of International Bargaining. En Evans, P.; Puntman, R. y Jacobson, H. Doubled Edge Diplomacy (pp. 3-43). Berkeley: California University Press.

Moravcsik, A. (1997). Taking Preferences Seriously: A Liberal Theory ofInternational Politics. International Organization, 513-553.

Morgenthau, H. (1993). Politics among Nations. The Struggle for Power and Peace. Nueva York: McGrawHill.

Neuman, S.G. (1998). International Relations Theory and the Third World. Nueva York: Saint Martin's Press.

Noesselt, N. (2014). China and Socialist Countries: Role Change and Role Continuity. GIGA Working Papers, 1-28.

Onuf, N. (1998). Constructivism: A User's Manual. En Kubálkova, V.; Onuf, N. y Kowert, P. (editores), International Relations in a Constructed World, (pp. 58-78). New York: M.E Sharpe.
Pachano, S. (2010). Ecuador: El nuevo sistema político en funcionamiento. Revista de Ciencia Política, $n^{\circ} 2,297-317$.

Paz y Miño Cepeda, J. J. (10-11/2006). Las elecciones presidenciales en Ecuador en 2006 y la propuesta de una Asamblea Constituyente. Historia y Presente, Taller de Historia Económica, Pontificia Universidad Católica del Ecuador.

Ponce Leiva, J. (2006). Las relaciones internacionales del Ecuador, desde 1979. AFESE, No. 66, 139-164.

Puntman, R. (1998). Diplomacy and domestic politics: the logic of two- level games. International Organization (verano), 427-460.

Ramírez, J. (2013). La politica migratoria en Ecuador: rupturas, continuidades y tensiones. Quito: Iaen.

Roche, J.-J. (1994). Le système international contemporain. París: Montchrestien.

Rosenau, J. (09/1968). Comparative Foreign Policy: Fad, Field or Fantasy? International Studies Quarterly $12, n^{\circ} 2,269-329$.

Russell, R. y Tokatlian, J.G. (12/2013). América Latina y su gran estrategia: entre la aquiescencia y la autonomía. Revista CIDOB d'Afers Internacionals, no $104,157-180$.

Russell, R. y Tokatlian, J.G. (2003). From Antagonistic Autonomy to Relational Autonomy: A Theoretical Reflection from the Southern Cone. Latin American Politics and Society 45, no 2 (primavera), $1-24$.

Rynning, S. y Guzzini, S. (2001). Realism and Foreign Policy Analysis. Working Paper, No. 72.

Schenoni, L. y Escudé, C. (2016). Peripheral Realism Revisited. Revista Brasileira de Politica Internacional, 1-18.

Smith, S. (2001). Foreign Policy is What States Make of it: Social Construction and International Relations Theory. En Kubálková, V. Foreign Policy in a Constructed World. Londres: M.E- Sharpe. 
Sterling-Folker, J. y Badie, D. (2012). Constructivism. En Hook, S. y Jones, C. Routledge Handbook of American Foreign Policy (pp. 104-113). Nueva York: Routlegde.

Thies, C. (2017). Role Theory and Foreign Policy Analysis in. Foreign Policy Analysis, 662-681.

Tickner, A. (2002). "Colombia" es lo que los actores estatales hacen de ella: une (re)lectura de la política exterior colombiana hacia Estados Unidos. En Ardila, M.; Cardona, D. y Tickner, A. Prioridades $y$ desafios de la politica exterior colombiana (pp. 353-397). Bogotá: Fescol.

Tickner, A. (12/2008). Latin American IR and the Primacy of lo práctico. International Studies Review 10, no $^{\circ}, 735-748$.
Waltz, K. (1979). Theory of International Politics. Boston: McGrawHill.

Wendt, A. (1992). Anarchy is what States Make of it: The Social Construction of Power Politics. International Organization, 391-425.

Wendt, A. (1999). Social Theory of International Politics. Cambridge: University Press.

Yeo, A. (2010). Ideas and Institutions in Contentious Politics: Anti-U.S. Base Movements in Ecuador and Italy. Comparative Politics, 435-455.

Zepeda, B. y Egas, M.G. (07-10/2011). La política exterior de la revolución ciudadana: opinión y actitudes públicas. Revista Mexicana de Política Exterior, 95-134. 